\title{
A WBAN System for Ambulatory Monitoring of Physical Activity and Health Status: Applications and Challenges
}

\author{
E. Jovanov ${ }^{1}$, A. Milenkovic ${ }^{1}$, C. Otto ${ }^{1}$, P. De Groen ${ }^{2}$, B. Johnson ${ }^{2}$, S. Warren ${ }^{3}$, and G. Taibi ${ }^{4}$ \\ ${ }^{1}$ Electrical and Computer Engineering, University of Alabama in Huntsville, Huntsville, Alabama, U.S.A. \\ ${ }^{2}$ Mayo Clinic, Rochester, Minnesota, U.S.A. \\ ${ }^{3}$ Electrical and Computer Engineering, Kansas State University, Manhattan, Kansas, U.S.A. \\ ${ }^{4}$ SmartWorlds, Boston, Massachusetts, U.S.A.
}

\begin{abstract}
Recent technological advances in sensors, lowpower integrated circuits, and wireless communications have enabled the design of low-cost, miniature, lightweight, intelligent physiological sensor platforms that can be seamlessly integrated into a body area network for health monitoring. Wireless body area networks (WBANs) promise unobtrusive ambulatory health monitoring for extended periods of time and near real-time updates of patients' medical records through the Internet. A number of innovative systems for health monitoring have recently been proposed. However, they typically rely on custom communication protocols and hardware designs, lacking generality and flexibility. The lack of standard platforms, system software support, and standards makes these systems expensive. Bulky sensors, high price, and frequent battery changes are all likely to limit user compliance.
\end{abstract}

To address some of these challenges, we prototyped a WBAN utilizing a common off-the-shelf wireless sensor platform with a ZigBee-compliant radio interface and an ultra low-power microcontroller. The standard platform interfaces to custom sensor boards that are equipped with accelerometers for motion monitoring and a bioamplifier for electrocardiogram or electromyogram monitoring. Software modules for on-board processing, communication, and network synchronization have been developed using the TinyOS operating system. Although the initial WBAN prototype targets ambulatory monitoring of user activity, the developed sensors can easily be adapted to monitor other physiological parameters. In this paper, we discuss initial results, implementation challenges, and the need for standardization in this dynamic and promising research field.

Keywords-Wireless Body Area Networks, Ambulatory Monitoring, Activity Monitors, Sensors

\section{INTRODUCTION}

Wireless body area networks (WBANs) signify emerging technology with the potential to revolutionize health care by allowing unobtrusive health monitoring for extended periods of time [1][2]. A typical WBAN consists of a number of inexpensive, lightweight, and miniature sensor platforms, each featuring one or more physiological sensors: motion sensors, electrocardiographs (ECGs), electromyographs (EMGs), and/or electroencephalographs (EEGs). The sensors could be located on the body as tiny intelligent patches, integrated into clothing, or implanted below the skin or muscles.

Monitoring physical activity has become increasingly important as many studies link physical activity with overall health status [3]. Solutions range from inexpensive devices such as pedometers to complex implementations featuring several accelerometers and gyroscopes located on different characteristic points on the body (ankle, arm, waist, thigh, etc.) $[3][4][5][6]$. Pedometers can help to provide a rather rudimentary measure of user activity by counting the number of steps during selected periods. More precise measurements can be achieved using a more complicated setup. However, interfacing these sensors is a challenge. Use of communication buses integrated into clothing is applicable only in some situations; we believe the unobtrusive solution of choice will be wireless body area networks of sensors. Although some research systems utilize on-sensor signal processing, most systems still rely on raw data transmission, where data are processed on an external computer or personal server. Increased sensor intelligence reduces the need for communication, therefore minimizing power consumption and extending battery life.

One class of the commercially available wearable systems allows recording of physiological data that are processed offline. The size and weight of these systems can interfere significantly with normal user behavior. Our experience with the development of wearable health monitors indicates that most critical system implementation issues cannot be predicted using simulations, or prototypes that are significantly different from the target system. On the other hand, custom developed wireless sensors are often unobtrusive and can capture natural activity patterns. However, a lack of standards and development environments increases the design time and system cost.

We are therefore developing a prototype system for ambulatory monitoring of user activity with off-the-shelf wireless sensor platforms and custom sensor modules. These platforms can be customized for individual users and an application-specific set of sensors then seamlessly integrated into a WBAN. A WBAN can be used for many applications, including prolonged ambulatory monitoring, computer-supervised rehabilitation, emergency medical care, and continual patient monitoring in hospitals [7][8]. 
This research has the following major goals:

- Prototype a WBAN system with unobtrusive, commercially available sensor platforms that have minimum size and weight (to resolve system implementation and everyday use issues).

- Develop algorithms to reliably monitor user activity with a minimal number of accelerometers (to reduce the price of future systems).

- Improve system reliability using synergy of WBAN information.

In this paper, we present initial system development and integration experiences. We also discuss major implementation issues and possible applications.

\section{METHODS}

We are developing a multi-tiered system for ambulatory monitoring of an individual's physical activity and heart or muscle activity. Tier 1 encompasses Activity Sensor (ActiS) platforms integrated into a WBAN. Tier 2 encompasses a personal server application running on a PDA, cell phone, or home personal computer. Tier 3 includes medical server(s) that are accessed via the Internet. Typical deployment configurations are given in Fig. 1. The configuration on the left is optimized for maximum battery life. Individual sensors communicate only with the personal server (PS) in a WBAN. The PS communicates with the Home Server (HS) using Wireless Local Area Network (WLAN). A HS is typically already connected to the Internet. This configuration is also applicable for inpatient monitoring. Ambulatory monitoring requires the direct connection of a PS to the Internet (WAN connectivity), as illustrated in the center configuration. Finally, a modification of the first configuration may exclude the PS to reduce the system price, as shown in the rightmost depiction, but this is likely to increase total power consumption.

Our initial WBAN consists of 3 ActiS sensor platforms: two attached to the ankles of both legs and one that monitors ECG activity and upper body tilt. The sensors communicate with the personal server via a network coordinator ( $\mathrm{NC}$ in Fig. 1). The ActiS sensor was developed specifically for WBAN-based, wearable monitoring and computer-assisted physical rehabilitation applications.

Each ActiS platform utilizes a standard Telos platform from Moteiv [9] and a custom intelligent signal processing daughter card, which is attached to the Telos platform [10]. The Telos platform is an ideal fit for this application due to its small footprint (size of two AA batteries) and open source system software support. The second-generation Telos platforms feature an $8 \mathrm{MHz}$ MSP430F1611 microcontroller with an integrated $10 \mathrm{~KB}$ of RAM and 48 KB of flash memory, a Universal Serial Bus (USB) interface for programming and communication, and an integrated wireless ZigBee-compliant radio with an onboard antenna. In addition, the Telos platform includes humidity, temperature, and light sensors that could be used for environmental sensing. The Telos platform features a 10-pin expansion connector with one UART (Universal Asynchronous Receiver Transmitter) and $\mathrm{I}^{2} \mathrm{C}$ interface, two

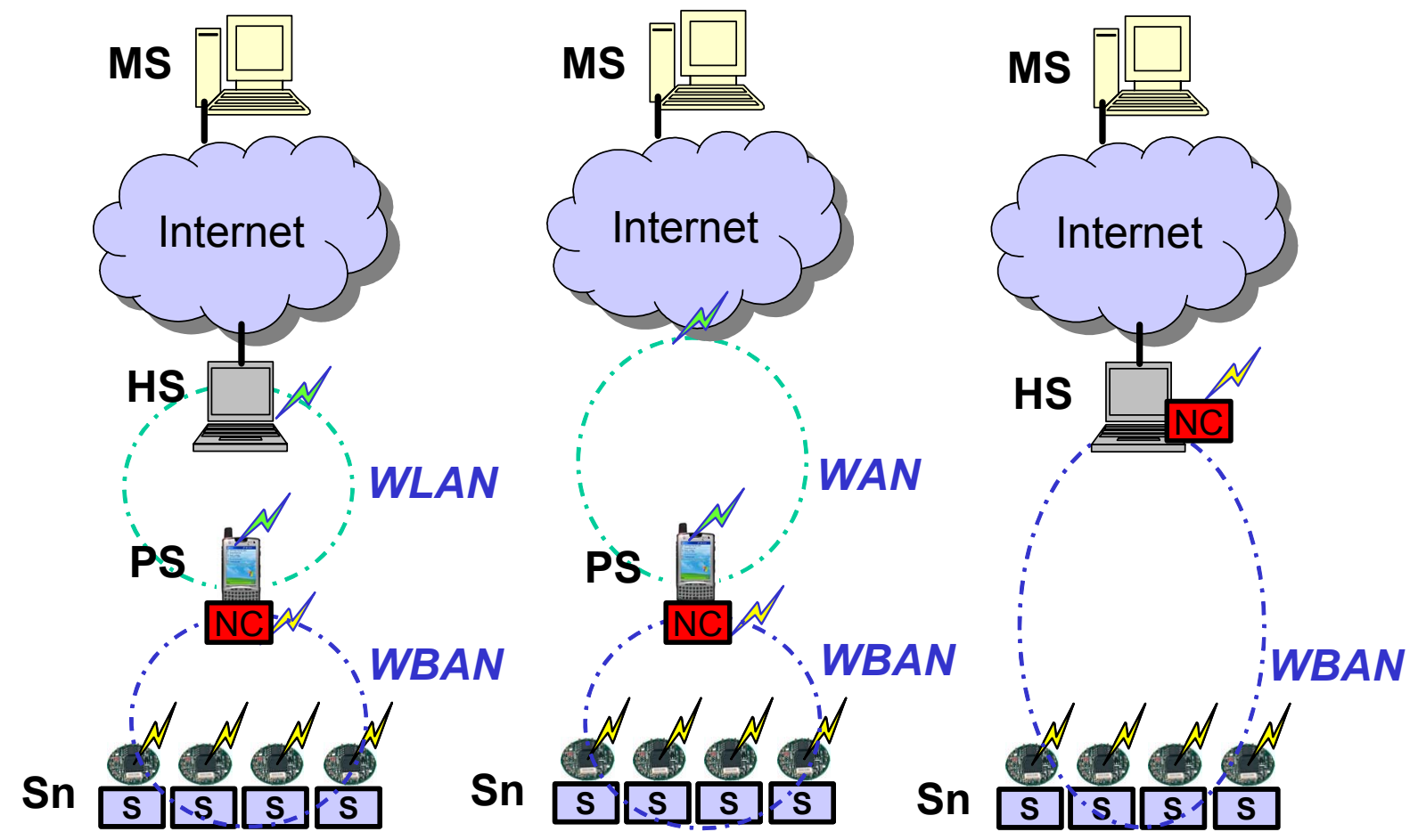

Fig. 1. WBAN configurations. Legend: Sn - Physiological sensors, WBAN - Wireless Body Area Network, PS - Personal Server, WLAN Wireless Local Area Network, WAN - Wide Area Network, HS - Home Server, MS - Medical Server, NC - WBAN network coordinator. 
general-purpose I/O lines, and three analog input lines.

Two custom boards, an IAS (Intelligent Activity Sensor) and an ISPM (Intelligent Signal Processing Module), have been developed as daughter boards for the Telos platform [10]. The ISPM board integrates a onechannel bio-amplifier and three accelerometer channels with a low power microcontroller, while the IAS board is a stripped down version of the ISPM with only accelerometer sensors and signal conditioning for a force sensing resistor that can be used as a foot switch. Fig. 2 shows the ISPM and IAS custom boards.

Both daughter cards utilize two perpendicular dual axis accelerometers (Analog Devices ADXL202) to cover three axes of motion. The sensor boards feature a MSP430F 1232 microcontroller for sampling and low-level data processing. This microcontroller was selected primarily for its compact size and ultra low power operation. The F1232 has a hardware UART that is used for communications with the Telos platform.

The user's physiological state is monitored using an onboard bio-amplifier implemented on the ISPM board. The bio-amplifier could be used for electromyogram (EMG) or electrocardiogram (ECG) monitoring. The output of the signal conditioning circuit is connected to the local microcontroller as well as to the microcontroller on the Telos board via the expansion connector. The A/D converter on the Telos board has a higher precision (12 bits) than the

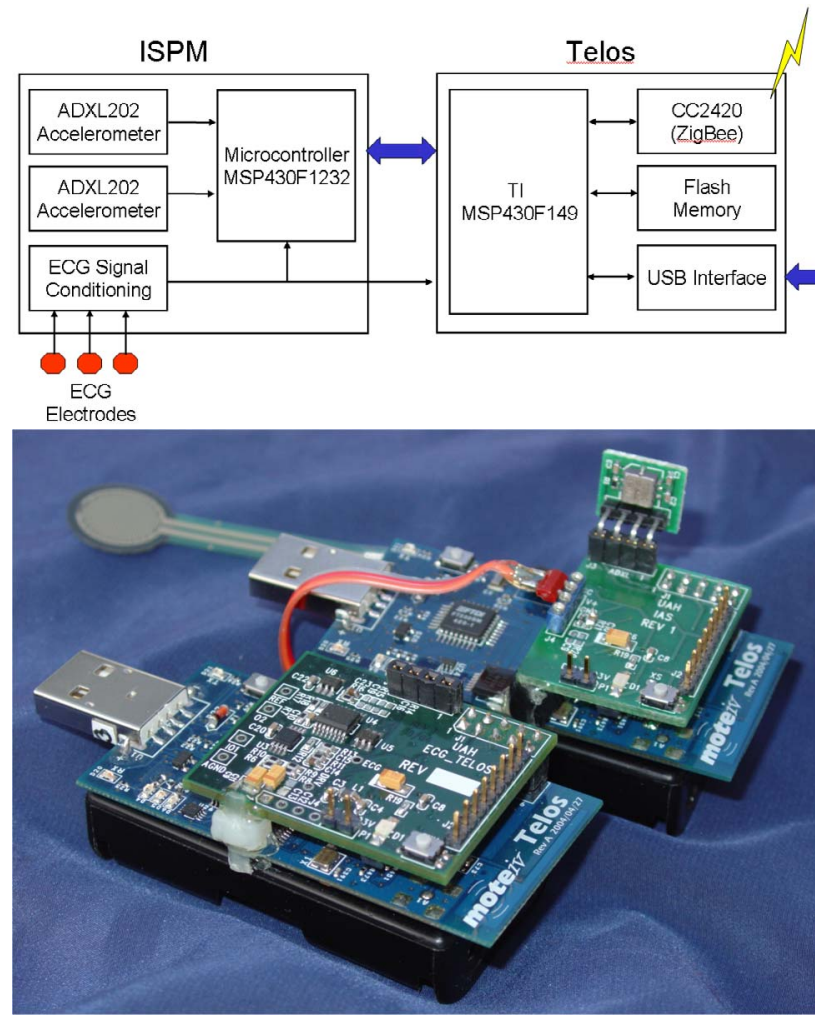

Fig. 2. ActiS sensor node. Block diagram (top); ActiS with ISPM (left) and IAS (right). A force sensing resistor and the second accelerometer are connected to the IAS board.
F1232 on the ISPM board (10 bits). This configuration provides the flexibility to utilize either one or both microcontrollers in parallel to process physiological signals.

The ActiS platform mounted on an ankle is used to recognize steps and measure stride and forces during walking or running. We plan to use those measurements in a number of computer assisted physical rehabilitation applications [7]. Low frequency components of acceleration signals represent projections of the gravity force $(\mathrm{g})$ on the accelerometer's reference axes. Rotations of the sensor in the vertical plane can be estimated as arctan of the ratio of horizontal and vertical acceleration components. Instead of calculating the angular position, many systems use offthe-shelf gyroscopes to measure angular velocity for the detection of gait phases [5]. However, a single accelerometer can significantly reduce the price of the sensor.

\section{RESULTS}

The WBAN has been successfully prototyped, and initial system software for network configuration, synchronization, data processing, and data transfer has been developed and tested. The main functions of the current WBAN prototype are as follows:

- Reset/Calibration/Synchronization/Session Initialization from the PS

- Runtime transfer of accelerometer and ECG data to the personal server

- Runtime on-board processing of accelerometer data for step detection; in this case only a time-stamped message is sent as an indication that a new step has occurred. Our first results indicate that a heuristic application-specific algorithm can reliably detect step phases with on-sensor processing.

- Synergy of information from different sensors. For example, stride is calculated from individually recognized steps on each leg sensor.

Another important issue is precise time synchronization, which is implemented in this WBAN. Even in the case of very infrequent messages ( 1 message every 2 seconds) our system is synchronized within half of a clock crystal cycle $(15 \mu \mathrm{s})[11]$.

Hierarchical processing has been employed to optimize power consumption and increase processing power. For example, a local processor on each daughter card performs preprocessing of accelerometer data (via a low pass filter and resampling), a processor on each sensor platform executes the step detection algorithm, while a processor on the personal server integrates information from all the sensors in the WBAN, maintains a user interface, and communicates with the remote medical server.

The sensors are carefully designed to maximize battery life. The ActiS board with the ISPM module draws $22.6 \mathrm{~mA}$ during wireless transmission, while the IAS board requires $22.4 \mathrm{~mA}$ [12]. Sensor intelligence allows event-driven 
messaging rather than constant transmission, which dramatically reduces power consumption. For example, monitoring of physical activity with a $1 \%$ wireless communication duty cycle achieves an ActiS average power consumption of only $8 \mathrm{~mW}$, allowing continuous monitoring without battery replacements for 31 days.

\section{Discussion AND CONCLUSIONS}

The initial test results for this WBAN prototype, as well as expected technological advances, indicate the tremendous potential of WBAN technology for ambulatory monitoring. Several emerging technologies, such as extremely low power wireless MEMS transceivers [13], promise further performance improvements of 2-3 orders of magnitude. However, a number of challenging tasks should be further addressed in an effort to make this technology affordable, robust, secure, and easy to wear. Further challenges include:

- Standards for wireless communication, messaging, and system support.

- Sensor type and precision.

- Sensor placement and mounting. Sensor attachment can create problems with spurious oscillations.

- Further sensor size reduction and ruggedization. Development of power efficient on-sensor signal processing algorithms and technological advances will eventually allow energy scavenging from the environment (e.g., the use of body movements to power activity sensors).

- Commodization of sensors. We believe the near-term target price should be less than $\$ 50$ per node.

- Security and privacy in WBAN and WAN communication. The ZigBee wireless interface on the Telos platform supports hardware message encryption that can be used to protect WBAN communication. Communication between a personal server and a medical server uses standard Internet security solutions.

- Seamless customization, configuration, and integration of sensors into a WBAN [14], and automatic uploads to support intermittent upload links to the medical server.

- Effective audio or visual user interfaces on the personal server. Given the increasing number of users familiar with the use of cell phones and PDAs, we expect wider user acceptance [15].

\section{ACKNOWLEDGMENTS}

The authors thank John Gober for his contribution to the ISPM and IAS printed circuit board development.

\section{REFERENCES}

[1] R.S.H. Istepanian, E. Jovanov, Y.T. Zhang, "Guest Editorial Introduction to the Special Section on M-Health: Beyond Seamless Mobility and Global Wireless Health-Care Connectivity," IEEE Transactions on Information Technology in Biomedicine, Dec. 2004, 8(4): 405 - 414

[2] D. Raskovic, T. Martin, E. Jovanov, "Medical Monitoring Applications for Wearable Computing," The Computer Journal, July 2004, 47(4): 495-504.

[3] B.G. Steele, B. Belza, K. Cain, C. Warms, J. Coppersmith, J. Howard, "Bodies in motion: Monitoring daily activity and exercise with motion sensors in people with chronic pulmonary disease," Journal of Rehabilitation Research \& Development, Sep/Oct 2003, Supplement 2, 40(5): $45-58$.

[4] S.W. Lee, K. Mase, "Activity and Location Recognition Using Wearable Sensors," Pervasive Computing, July-Sep. 2002, 1(3): 24 32.

[5] I.P.I. Pappas, T. Keller, S. Mangold, M.R. Popovic, V. Dietz, M. Morari, "A Reliable Gyroscope-Based Gait-Phase Detection Sensor Embedded in a Shoe Insole," IEEE Sensors Journal, Apr 2004, 4(2): 268-274.

[6] A. Krause, D.P. Siewiorek, A. Smailagic, J. Farringdon, "Unsupervised, Dynamic Identification of Physiological and Activity Context in Wearable Computing," Proc. 7th International Symposium on Wearable Computers, White Plains, NY, 2003, pp. 88-97.

[7] E. Jovanov, A. Milenkovic, C. Otto, P. C. de Groen, "A wireless body area network of intelligent motion sensors for computer assisted physical rehabilitation," Journal of NeuroEngineering and Rehabilitation, 2:6, March 1, 2005. [http://www.jneuroengrehab.com/content/2/1/6]

[8] D. Malan, T.R.F. Fulford-Jones, M. Welsh, S. Moulton, "CodeBlue: An Ad Hoc Sensor Network Infrastructure for Emergency Medical Care," Proc. of the MobiSys 2004 Workshop on Applications of Mobile Embedded Systems (WAMES 2004), Jun 2004, pp. 12-14.

[9] Moteiv, http://www.moteiv.com.

[10] C. Otto,. J.P. Gober, R.W. McMurtrey, A. Milenković, E. Jovanov, "An Implementation of Hierarchical Signal Processing on Wireless Sensor in TinyOS Environment," Proc. of the 43rd Annual ACM Southeast Conference ACMSE 2005, Vol. 2, Kennesaw, Georgia, March 2005, pp. 49-53.

[11] D. Cox, E. Jovanov, A. Milenkovic, "Time Synchronization for ZigBee Networks," Proc. of the $37^{\text {th }}$ IEEE Southeastern Symposium on System Theory (SSST'05), Tuskegee, AL, March 2005, pp. 135138.

[12] A. Milenkovic, M. Milenkovic, E. Jovanov, D. Hite,"An Environment for Runtime Power Monitoring of Wireless Sensor Network Platforms," Proc. of the $37^{\text {th }}$ IEEE Southeastern Symposium on System Theory (SSST'05), Tuskegee, AL, March 2005, pp. 406-410.

[13] B.P. Otis, JM Rabaey, "A 300- $\mu \mathrm{W}$ 1.9-GHz CMOS Oscillator Utilizing Micromachined Resonators," IEEE Journal of Solid-State Circuits, July 2003, 38(7): 1271-1274.

[14] S. Warren, "Beyond Telemedicine: Infrastructures for Intelligent Home Care Technology," Pre-ICADI Workshop on Technology for Aging, Disability, and Independence, The Royal Academy of Engineering, Westminster, London, England, June 2003.

[15] E. Bielli, F. Carminati, S. La Capra, M. Lina, C. Brunelli, M. Tamburini, "A Wireless Health Outcomes Monitoring System (WHOMS): development and field testing with cancer patients using mobile phones," BMC Medical Informatics and Decision Making, 2004, 4:7, http://www.biomedcentral.com/1472-6947/4/7 